\title{
Syntaphilin Is a Novel Biphasic Biomarker of Aggressive Prostate Cancer and a Metastasis Predictor
}

Michael J. Hwang, ${ }^{*}$ Kelly G. Bryant, ${ }^{\dagger}$ Jae H. Seo, ${ }^{\dagger}$ Qin Liu, ${ }^{\dagger}$ Peter A. Humphrey, ${ }^{\star}$ Mary Ann C. Melnick, ${ }^{\ddagger}$ Dario C. Altieri, ${ }^{\dagger}$ and Marie E. Robert*

From the Department of Pathology, ${ }^{*}$ Yale School of Medicine, New Haven, Connecticut; the Immunology, Microenvironment and Metastasis Program, ${ }^{\dagger}$ The Wistar Institute, Philadelphia, Pennsylvania; and the Yale Comprehensive Cancer Center, ${ }^{\ddagger}$ Yale School of Medicine, New Haven, Connecticut

Accepted for publication

February 20, 2019.

Address correspondence to Marie E. Robert, M.D., Department of Pathology, Yale School of Medicine, 310 Cedar St., PO Box 208023, New Haven, CT 06520. E-mail: marie.robert@yale.edu.

\begin{abstract}
Easily accessible biomarkers that may inform on the metastatic potential of localized prostate cancer are urgently needed. Herein, we show that syntaphilin (SNPH), a molecule originally identified as a negative regulator of mitochondrial dynamics in neurons, is abundantly expressed in prostate cancer. SNPH distribution in prostate cancer is spatially biphasic, with high expression at the invasive front, correlating with increased proliferative rates, as determined by Ki-67 labeling, and reduced levels in the central tumor bulk, which are further decreased in patients with distant metastases. Higher levels of SNPH are observed with increasing Gleason grade. Prostate tumors predominantly express a novel, extraneuronal isoform of SNPH that accumulates in mitochondria and maintains oxidative metabolism and tumor cell proliferation. These data suggest that SNPH is a novel marker of high Gleason grade prostate cancer, differentially expressed at the invasive front compared with the central tumor bulk, and is potentially down-regulated in metastatic disease. This biphasic pattern of expression may reflect a dual function of SNPH in controlling the balance between cell proliferation and invasion in tumors. (Am J Pathol 2019, 189: 1180-1189; https://doi.org/10.1016/j.ajpath.2019.02.009)
\end{abstract}

Prostate cancer remains the most prevalent malignancy diagnosed in men and the second leading cause of cancerassociated mortality in the Western world. ${ }^{1}$ Most prostate cancer cases are not lethal as slow-growing cells remain localized in the gland, and many patients are potentially best managed by active surveillance rather than aggressive treatments carrying significant morbidity. ${ }^{2}$ The 5-year survival rate of these patients approaches $100 \%$ but decreases to $29 \%$ to $30 \%{ }^{3,4}$ in men whose disease has spread beyond the prostate.

Morphologically indistinguishable from clinically localized high-grade disease, metastatic prostate cancer exhibits monoclonal origin in primary multifocal lesions, ${ }^{5}$ crossmetastatic seeding, ${ }^{6,7}$ and aggressive evolution in response to androgen-deprivation therapy. ${ }^{8}$ Despite considerable effort, the cellular and molecular requirements of metastatic prostate cancer have remained elusive, and there are currently no reliable approaches to identify the subset of prostate cancer patients who will eventually acquire metastatic competence and disseminated disease.

Recent experimental evidence has underlined the importance of tumor metabolism in disease progression. Considerable interest has been devoted to the bioenergetics shift toward aerobic glycolysis observed in most tumors, including prostate cancer, the so-called Warburg effect. ${ }^{9}$ However, more recent data have demonstrated that mitochondrial bioenergetics continue to play an important role in tumor metabolism, conferring malignant traits of drug resistance, tumor repopulation, and metastatic competence. ${ }^{10-13}$ The molecular determinants of this process are only beginning to emerge, but there is evidence that changes in mitochondrial dynamics, a process that affects organelle size, shape, and subcellular

Supported by NIH grants R35 CA220446 and P01 CA140043 (D.C.A.). Disclosures: None declared. 


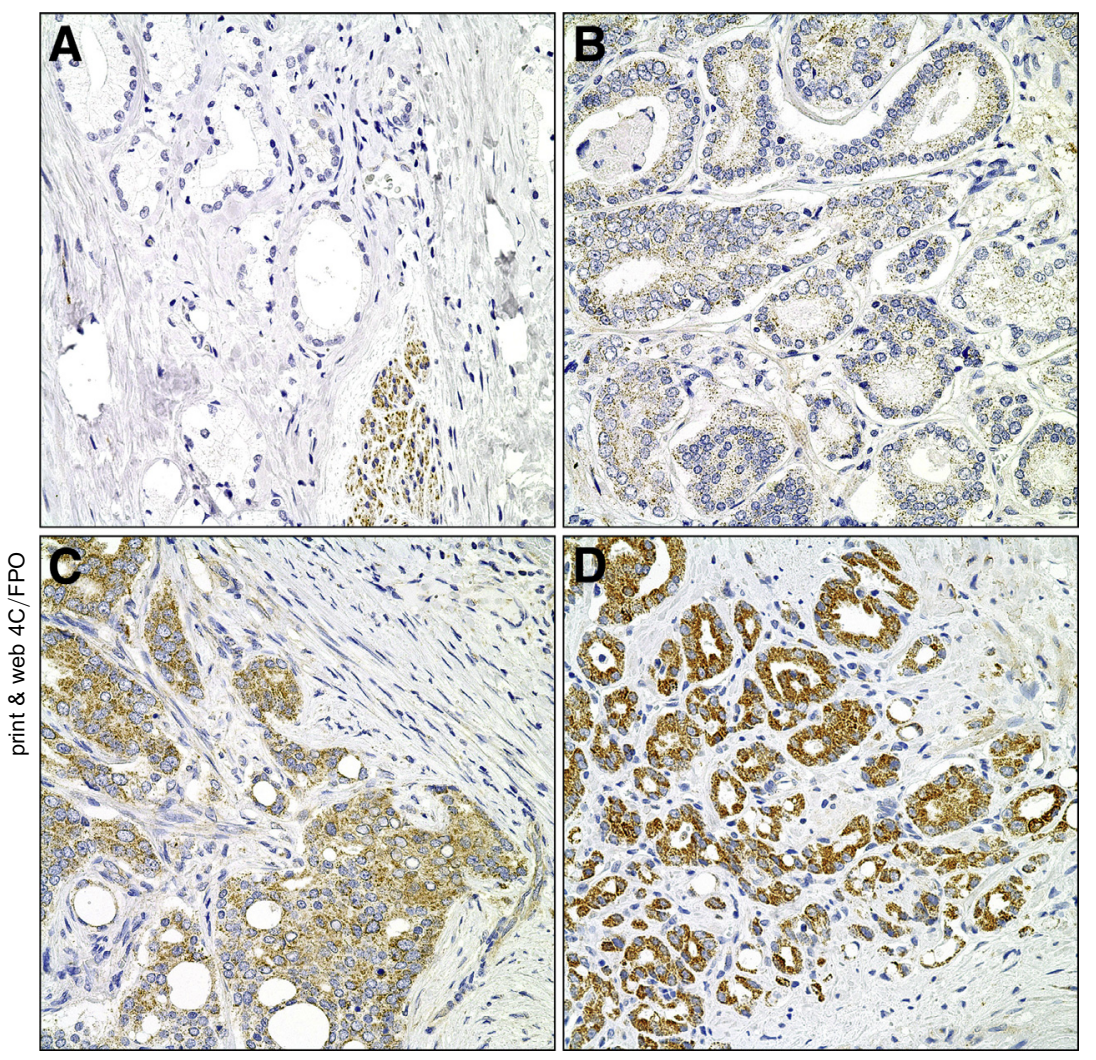

Figure 1 Characteristics and scoring method of SNPH immunohistochemical stain. A: Score 0: Gleason pattern (GP) 3 cancer cells with negative staining. A nerve bundle at the right lower corner serves as an internal positive control and demonstrates granular cytoplasmic positivity. B: Score 1+: GP 3 cancer cells demonstrating finely granular, loosely spaced cytoplasmic reactivity. C: Score 2+: GP 4 tumor cells showing granular intracytoplasmic reactivity occupying a greater proportion of cytoplasmic space than a score of $1+$, but less than that seen in $3+$ in both quantity and quality of the stain. D: Score $3+$ : Bright and closely packed intracytoplasmic granular staining that occupies the entire cytoplasm of most tumor cells and occasionally obscures the nuclei. Original magnification, $\times 400$ (A-D). motility, regulate tumor cell invasion and metastatic dissemination in mouse models of disease. ${ }^{14}$

In this context, a molecule originally described as a negative regulator of mitochondrial dynamics in neurons, ${ }^{15}$ syntaphilin (SNPH), has been identified as a key determinant of the balance between cell proliferation and cell invasion in tumors, including prostate cancer. ${ }^{16}$ Regulated by stress stimuli of the tumor microenvironment, high levels of SNPH maintain mitochondrial oxidative bioenergetics and buffer reactive oxygen species to support tumor cell proliferation. Conversely, unfavorable conditions of the microenvironment, such as hypoxia, result in decreased expression of SNPH in tumors. In turn, this lowers tumor cell proliferation but promotes the redistribution of energetically active mitochondria to the cortical cytoskeleton, fueling increased tumor cell motility, invasion, and metastatic dissemination, in vivo. ${ }^{14,16}$ Analysis of genomic databases suggests that SNPH expression is decreased or lost in advanced malignancies; thus, it is in line with greater metastatic propensity. ${ }^{14}$ However, a potentially biphasic function of SNPH at the cell proliferation-cell invasion interface in primary patient samples has not been previously investigated.

In this study, we examined the expression of SNPH in a cohort of localized and metastatic prostate cancer patients. SNPH levels were found to be spatially controlled, and low levels of expression in the central tumor bulk were associated with metastatic disease.

\section{Materials and Methods}

\section{Patient Cohort}

On approval from the Yale University (New Haven, CT) Institutional Review Board, the archival database of the Department of Pathology at Yale New Haven Hospital (New Haven, CT) was searched for radical prostatectomy specimens removed to treat prostatic adenocarcinoma between 2007 and 2016. Hematoxylin and eosin-stained sections of tumor from each specimen were reviewed by two pathologists (M.J.H. and M.E.R.), and a total of 89 prostatic adenocarcinoma cases were selected ( 88 radical prostatectomy and 1 cystoprostatectomy specimens). The 2015 modified International Society of Urological Pathology Gleason Scheme was used to assign Gleason pattern (GP) and Gleason scores (GSs), and cases were furthered categorized into five grade groups (GGs), according to the grade group system endorsed in the 2016 World Health Organization classification system. ${ }^{17,18}$ Tumor stage was assigned according to the American Joint Committee on Cancer TNM (tumor, node, metastasis) classification, seventh edition. ${ }^{19}$ To compare SNPH expression levels between indolent and potentially aggressive disease, 29 cases of GS 6 (GG 1) and 60 cases of GS 7 to 10 (GG 2 to 5 ) were selected. Sixteen of the GG 2 to 5 patients had synchronous or metachronous regional lymph node or distant metastases. For all patients, the tissue block containing the highest volume of tumor and, in the case of GS 

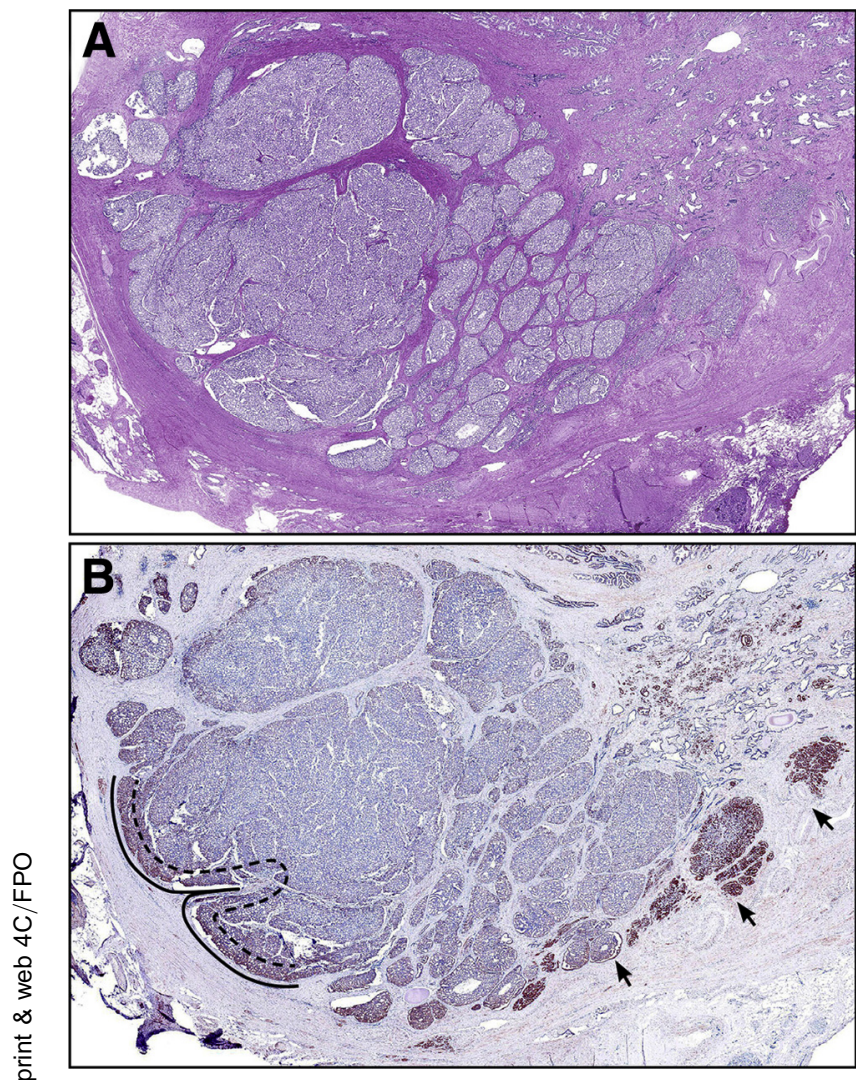

Figure 2 A: Low-magnification view of a cancer nodule, with the central tumor bulk consisting predominantly of invasive cribriform Gleason pattern 4 (hematoxylin and eosin stain). B: Low-magnification view of the same cancer nodule, with an outer rim of accentuated SNPH staining. The invasive front is defined as the external rim of each cancer nodule abutting the adjacent stroma, as outlined in this image as the zone between the dotted and solid lines. Arrows denote accentuated SNPH staining at the tumor-stromal interface. Original magnification $\times 12.5$ (A and B).

7 to 10 , the highest percentage of GP $4 / 5$ was selected for immunohistochemical analysis. All cases were subjected to SNPH analysis (see below). A subset was additionally evaluated by Ki-67 immunostaining.

In addition to primary tumor assessment, SNPH expression was evaluated in paired metastases from 15 of the 16 patients with metastases in whom tissue from the metastases was available for staining (14 with regional lymph node metastases, 1 of whom also had a distant metastasis, and 1 with three separate distant metastases). SNPH expression was also assessed in 15 additional unpaired distant metastases.

\section{Immunohistochemistry}

For SNPH analysis, sections ( $4 \mu \mathrm{m}$ thick) were incubated for 1 hour with a rabbit polyclonal antibody against SNPH (number HPA049393; Sigma, St. Louis, MO) at 1:300 dilution. Antibody reactivity was detected with a kit using peroxidase-diaminobenzidine as the chromogen (DAB UltraView; Ventana, Tucson, AZ), and all slides were counterstained with hematoxylin. A negative control was run with each batch of slides without applying the primary antibody. Immunostaining for Ki-67 was performed on sections ( $4 \mu \mathrm{m}$ thick) and run on the Ventana Benchmark XT automated stainer with the monoclonal MIB-1 (Dako, Carpenteria, CA) antibody at 1:300 dilution.

\section{Pathology Review and Scoring}

SNPH stained slides were scored simultaneously by two pathologists (M.J.H. and M.E.R.). For each slide, the percentage of cell staining and the intensity of SNPH stain in cancer cells were evaluated and recorded, both by GP and location in the gland. That is, each GP component present on a slide was separately scored for SNPH on a 0 to $3+$ semiquantitative scale, as follows: 0 indicates no staining; $1+$, faint, loosely spaced, fine granular cytoplasmic staining; $2+$, intermediate intensity between $1+$ and $3+$; and $3+$, bright, closely packed, coarse granular cytoplasmic staining frequently occupying the entire cytoplasm (Figure 1). Since cribriform pattern was first recognized as an independent prognostic factor for biochemical recurrence in $2011,{ }^{20}$ the adverse prognostic impact of the presence of cribriform pattern has been reported in association with postoperative metastasis, disease-specific survival, ${ }^{21}$ and adverse pathologic findings. ${ }^{22} \mathrm{~A}$ recent review article by Iczkowski et $a^{23}$ summarized the clinical significance of cribriform pattern in prostate cancer. Therefore, cribriform pattern was specifically scored in addition to other GP 4 as part of the association of SNPH with different, outcome-relevant prostate cancer pathologies. In addition, staining intensity was scored by tumor location, as follows: i) overall SNPH score in all tumor present on the slide; ii) the invasive front, defined as the external rim of a tumor nodule abutting benign tissue; iii) the central tumor bulk, defined as the central core of tumor, excluding the invasive front; and iv) staining in areas of extraprostatic extension (Figure 2). Invasive front and central tumor bulk scores were calculated for each tumor nodule present on the slide (with exception of eight GG 1 patients in whom the amount of tumor present was insufficient to calculate differential invasive front and central bulk score). $\mathrm{H}$ scores for each score (both by GP and location) were calculated as follows: 0 (percentage of cells with 0 intensity) +1 (percentage of cells with $1+$ intensity) +2 (percentage of cells with $2+$ intensity) +3 (percentage of cells with $3+$ intensity). For lymph node and distant metastases, the percentage of cell staining and intensity of SNPH stain in cancer cells were recorded.

After reviewing SNPH immunostains, 15 GG 2 to 5 cases, manifesting high SNPH expression at the invasive front relative to the central tumor bulk, were selected for proliferation index analysis by Ki-67 immunostain. For this analysis, two Ki-67 hot spots were identified at $\times 200$ magnification in both the invasive front and central tumor bulk locations. The number of Ki-67-positive nuclei within each location was counted on printed color images taken at $\times 400$ magnification. The Ki-67 labeling index (Ki-67 LI) was defined as the number of Ki-67-positive cancer cells divided by the total number of cancer cells present in the hot spot. 
Table 1 Demographic Data

\begin{tabular}{|c|c|c|c|}
\hline Variable & $\begin{array}{l}\text { Grade group } 1 \\
(n=29)\end{array}$ & $\begin{array}{l}\text { Grade group } 2 \\
\text { to } 5(n=60)\end{array}$ & $P$ value \\
\hline \multicolumn{4}{|l|}{ Age, years } \\
\hline Mean (SD) & $60.9(7.5)$ & $62.5(6.8)$ & 0.319 \\
\hline Median (range) & $61.0(46-81)$ & $63(48-75)$ & \\
\hline \multicolumn{4}{|l|}{$\begin{array}{l}\text { Preoperative PSA, } \\
\mathrm{ng} / \mathrm{mL}\end{array}$} \\
\hline Mean (SD) & $6.0(3.2)$ & $8.9(4.7)$ & 0.032 \\
\hline Median (range) & $5.5(0.9-13)$ & $7.6(3-23)$ & \\
\hline \multicolumn{4}{|c|}{ Cancer involvement, \% } \\
\hline Mean (SD) & $6.1(5.9)$ & $27.2(20.2)$ & $<0.001$ \\
\hline Median (range) & $5.0(1-30)$ & $20(5-90)$ & \\
\hline \multicolumn{4}{|l|}{ Grade group, $n(\%)$} \\
\hline 1 & $29(100)$ & $0(0)$ & $<0.001$ \\
\hline 2 & $0(0)$ & $7(11.7)$ & \\
\hline 3 & $0(0)$ & $25(41.7)$ & \\
\hline 4 & $0(0)$ & $4(6.6)$ & \\
\hline 5 & $0(0)$ & $24(40)$ & \\
\hline \multicolumn{4}{|l|}{ T stage, $n(\%)$} \\
\hline $\mathrm{T} 2$ & $28(96.6)$ & $20(33.3)$ & $<0.001$ \\
\hline T3 & $1(3.4)$ & $40(66.7)$ & \\
\hline \multicolumn{4}{|l|}{$\mathrm{N}$ stage, $n(\%)$} \\
\hline NO & $13(100)$ & $41(73.2)$ & 0.056 \\
\hline N1 & $0(0)$ & $15(26.8)$ & \\
\hline \multicolumn{4}{|l|}{$\begin{array}{l}\text { Seminal vesicle } \\
\quad \text { invasion, } n(\%)\end{array}$} \\
\hline Absent & $29(100)$ & $41(68.3)$ & $<0.001$ \\
\hline Present & $0(0)$ & $19(31.7)$ & \\
\hline \multicolumn{4}{|c|}{$\begin{array}{l}\text { Extraprostatic } \\
\quad \text { extension, } n(\%)\end{array}$} \\
\hline Absent & $28(96.6)$ & $22(36.7)$ & $<0.001$ \\
\hline Present & $1(3.4)$ & $38(63.3)$ & \\
\hline \multicolumn{4}{|l|}{ Margin, $n(\%)$} \\
\hline Negative & $27(93.1)$ & $32(54.2)$ & $<0.001$ \\
\hline Positive & $2(6.9)$ & $27(45.8)$ & \\
\hline \multicolumn{4}{|c|}{$\begin{array}{l}\text { Biochemical } \\
\quad \text { recurrence, } n(\%)\end{array}$} \\
\hline Absent & $28(100)$ & $34(60.7)$ & $<0.001$ \\
\hline Present & $0(0)$ & $22(39.3)$ & \\
\hline
\end{tabular}

$\mathrm{N}$, node; PSA, prostate-specific antigen; $\mathrm{T}$, tumor.

\section{SNPH RNA Expression}

Fresh tissue was collected prospectively from six radical prostatectomy specimens, to include both benign and malignant tissue (10 samples total). The collected specimens were bisected, with half snap frozen for RNA analysis and half submitted in formalin for routine histology to confirm tissue content. Flash-frozen human prostate tissue specimens were pulverized into a powder using a cold mortar and pestle. RNA was extracted from the tissue powder using the RNeasy Mini Kit (catalog number 74104; Qiagen, Redwood City, CA) and quantified on a Nanodrop (Thermo Fisher Scientific, Waltham, MA). cDNA was synthesized using $3 \mu \mathrm{g}$ of extracted RNA. The differential expression of $S N P H$ isoforms in prostate cancer samples was quantified by quantitative PCR, as described previously. ${ }^{16}$ Briefly, custom isoform-specific
TaqMan gene expression assays to detect long human $S N P H$ transcript (L-SNPH; https://www.ncbi.nlm.nih.gov; accession number NM_001318234.1) or short human SNPH transcript (S-SNPH; https://www.ncbi.nlm.nih.gov; accession number NM_014723) were used. Custom $L-S N P H$ assay primers and probes were as follows: forward, 5'-TCAGGGTTGTTGAGAGGAGTCA-3'; reverse, 5'-CCAGTTGGCCCGTGGTT- ${ }^{\prime}$; and probe, $5^{\prime}$-ATAATACGGGAAGCCCC-3' ${ }^{\prime}$. Custom $S-S N P H$ assay primers and probe were as follows: forward, 5'-AGTGGTGCGAGCCG-3'; reverse, 5' GGTGGGATGGGCGGTATC-3'; and probe, 5'-CAGTGGACTCAGCCCCC-3'. A standard synthetic gBlock containing the target amplicons for common SNPH (short + long), short SNPH, long SNPH, actin, and GAPDH in tandem was purchased from Integrated DNA Technologies (Coralville, IA). The efficiency of amplification of the three assays (long, short, and common SNPH) was determined using the $\mathrm{Ct}$ slope method, with six concentrations of common SNPH (short + long), short SNPH, long SNPH, actin, and GAPDH in tandem standard covering a 5-log range and found to be identical $(P>0.05$ for all comparisons). The means $\pm \mathrm{SD}$ were as follows: $\mathrm{Ex}$ long $=82.33 \pm 8.069$; Ex short $=80.90 \pm 2.700$; and $\mathrm{Ex}$ common $=84.82 \pm 7.179$. The means $\pm \mathrm{SD}$ for the correlation coefficients were: $R^{2}$ long $=0.9975 \pm$ $0.002500 ; R^{2}$ short $=0.9875 \pm 0.01250 ;$ and $R^{2}$ common $=0.9970 \pm 0.001000$. All $R^{2}$ values were identical $(P>0.05$ for all comparisons). Absolute copy number for each transcript was determined against a standard curve of common SNPH (short + long), short SNPH, long SNPH, actin, and GAPDH in tandem that was run in parallel with the cDNA samples. For relative quantitation, the $\Delta \Delta \mathrm{Ct}$ method was used.

\section{Statistical Analysis}

The association between categorical clinicopathologic features and grade group were evaluated by $\chi^{2}$ or Fisher's exact test. The means of continuous demographic data between grade groups were compared using a two-group $t$-test. A paired $t$-test was used to compare the $\mathrm{H}$ score of SNPH and Ki-67 LI between invasive front and central tumor bulk. A two-sample $t$-test was used to compare the $\mathrm{H}$ score of SNPH between GG 1 cases and GG 2 to 5 cases. One-way analysis of variance with post-hoc Bonferroni's correction was used to conduct multiple comparisons of $\mathrm{H}$ scores between different GPs. Cuzick's test was used for examing the trend of SNPH H scores over the ordered GPs. Two-sided tests were used for all analyses, with $P<0.05$ regarded as statistically significant.

\section{Results}

\section{Clinical and Pathologic Features of Patients}

Consistent with previous observations, significant differences in clinicopathologic features and outcome were observed in 

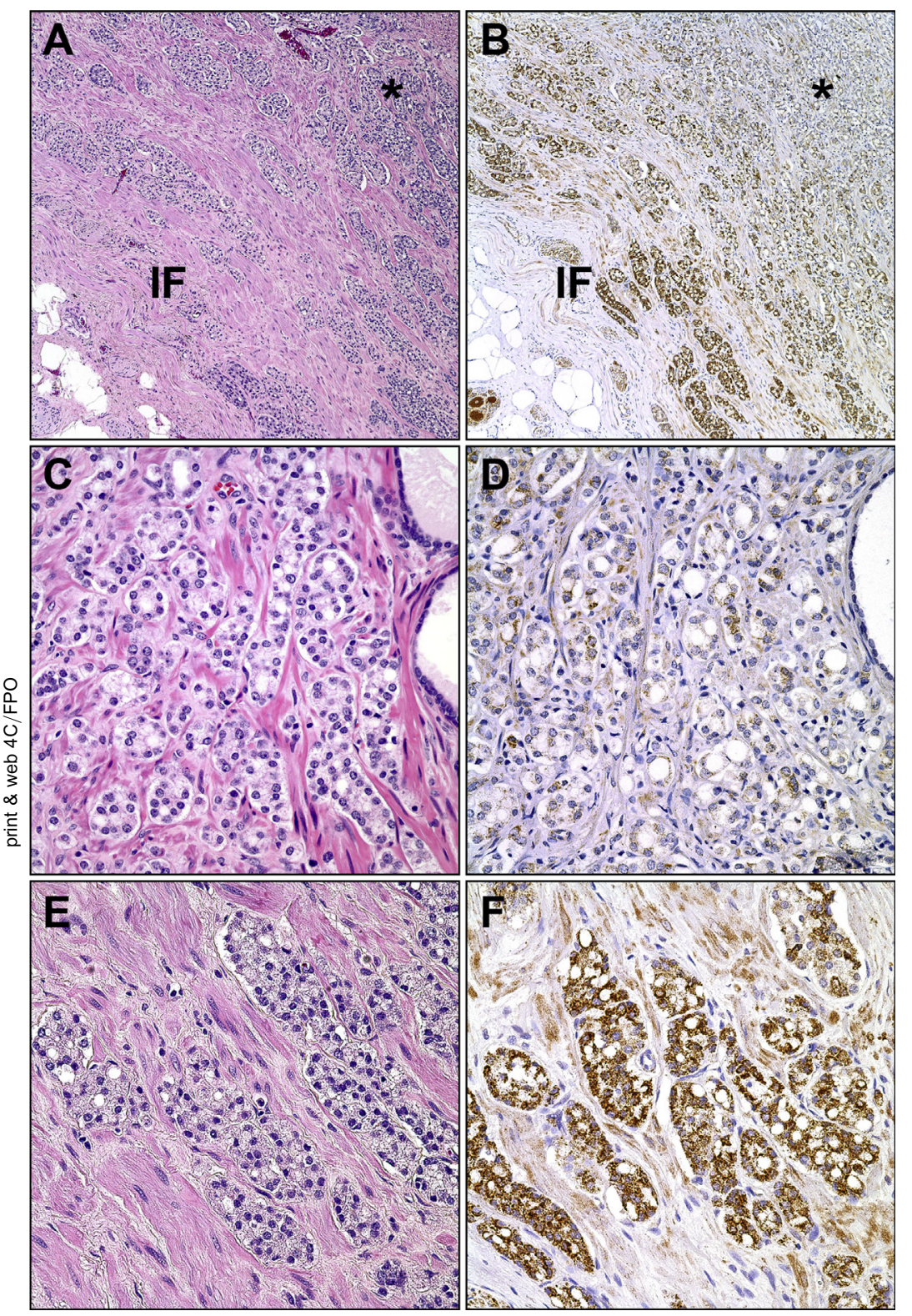

Figure 3 Hematoxylin and eosin-stained slides and corresponding SNPH immunohistochemical stains. A and B: Low-magnification view of the edge of a tumor nodule, with the invasive front (IF) at the left lower corner. The tumor cells are arranged in fused/poorly formed glands with accentuation of SNPH staining at the invasive front and gradual attenuated expression toward the central tumor bulk at the right upper corner (asterisks). C and D: Tumor cells at the central tumor bulk with weak, finely granular 0 to $1+$ positivity within the cytoplasm. $\mathbf{E}$ and $\mathbf{F}$ : Tumor cells at the invasive front, showing much higher $3+$ SNPH staining with coarse, closely packed cytoplasmic positivity. The granular positivity occupies the entire cytoplasm in most tumor cells. Original magnification: $\times 100(\mathbf{A}$ and $\mathbf{B}) ; \times 400(\mathbf{C}-\mathbf{F})$. the GG 1 and GG 2 to 5 patient groups ${ }^{24,25}$ (Table 1). GG 2 to 5 patients included 15 with synchronous lymph node metastasis, 1 of whom later developed a distant metastasis, and 1 with metachronous distant metastases. No differences in clinicopathologic features were found between patients with or without metastasis, except for the finding that the presence of extraprostatic extension was significantly associated with metastasis $(P=0.032)$ compared with no extraprostatic extension (Supplemental Table S1).

\section{Syntaphilin Immunohistochemistry Staining}

\section{Adenocarcinoma}

Immunohistochemical detection of SNPH in archival prostate cancer specimens was feasible and appeared as variably packed, faint to coarse granular cytoplasmic staining
(Figure 1). In the vast majority of tumors (78/81, 96.3\%), SNPH H scores were significantly higher at the invasive front compared with central tumor regions $(P<0.0001)$ (Figure 3, and Figure 4, A and B). In two cases, invasive front and central tumor $\mathrm{H}$ scores were identical; and in one case, the central tumor score was greater. In addition, invasive front $\mathrm{H}$ scores of GG 2 to 5 tumors were higher than the invasive front scores of GG 1 tumors $(P=0.008)$ (Figure 4C). No difference between central tumor $\mathrm{H}$ scores was found between GG 1 and GG 2 to 5 tumors. There was an increasing trend in SNPH expression with increasing GP $(P=0.001$ from Cuzick's test for trend) (Figure 4D). Higher SNPH H scores were observed with increasing GP, with GP $5 \mathrm{H}$ scores being significantly higher than GP 4 $(P<0.001)$ and GP $3(P=0.001) \mathrm{H}$ scores. GP $3 \mathrm{H}$ scores in GG 2 to 5 tumors were significantly higher than GP 3 

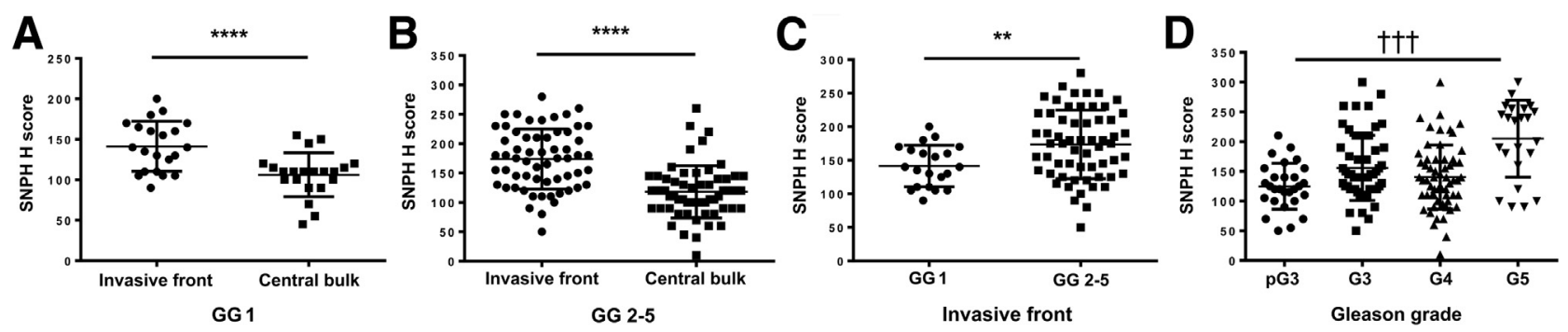

Figure 4 A and B: SNPH expression is higher at the invasive front than in central tumor bulk regions in both grade group (GG) 1 and GG 2 to 5 tumors. C: SNPH expression at the invasive front is higher in GG 2 to 5 tumors than in GG 1 tumors. D: There is a trend of higher SNPH expression in high-grade Gleason pattern (GP), with pure GP 3 in GG 1 demonstrating the lowest $\mathrm{H}$ score (pG3: pure Gleason pattern 3 in grade group 1; G3: Gleason pattern 3 in grade group 2-5; G4: Gleason pattern 4; G5: Gleason pattern 5). ${ }^{* *} P<0.01,{ }^{* * *} P<0.0001$ (paired $t$-test); ${ }^{\dagger \dagger \dagger} P<0.001$ (Cuzick trend test).

scores in GG 1 tumors $(P=0.004)$ (Figure 4D). Interestingly, extraprostatic extension $\mathrm{H}$ scores were greater than both paired invasive front $(P=0.017)$ and paired central tumor bulk $(P<0.001) \mathrm{H}$ scores (Supplemental Figure S1).

Considering cribriform pattern (G4C), in 60 patients with GG 2 to 5 scores, 31 (57\%) had a component of cribriform pattern 4. There was no difference in $\mathrm{H}$ scores for G3, G4, and G5 patterns between prostates with $(n=31)$ and without $(n=29)$ cribriform pattern $4(P=0.36$ to $P=0.95$ ). In addition, G4C $\mathrm{H}$ scores were no different (not greater) than $\mathrm{G} 4 \mathrm{H}$ scores in cases without a cribriform component $(P=0.97)$. Furthermore, when comparing $\mathrm{H}$ scores within the same tumor, $\mathrm{G} 4 \mathrm{C} \mathrm{H}$ scores were not greater than scores in any other Gleason pattern, whereas G5 $\mathrm{H}$ scores were significantly higher than G4C scores $(P=0.048)$.

Central tumor SNPH H scores were significantly lower in the 16 patients with synchronous or metachronous metastases compared with central $\mathrm{H}$ scores in patients without metastases $(P=0.027)$ (Figure 5). By contrast, invasive front $\mathrm{H}$ scores were not different in patients with and without metastasis. SNPH H scores did not correlate with T stage or evidence of biochemical recurrence. Most distant metastases expressed SNPH strongly, with greater intensity than that found in either regional lymph node metastases $(P=0.015)$ or primary tumors $(P<0.001)$ (Supplemental Figure S2).

\section{Benign Prostate}

SNPH staining was present in benign tissue in all samples, with a variety of staining intensities noted (Figure 6). Glands in the peripheral and central zones typically showed weak, powdery cytoplasmic staining $(1+)$. The ejaculatory ducts and seminal vesicles stained strongly with coarse intracytoplasmic granules ( 2 to $3+$ ). Normal urothelium or urothelial metaplasia typically demonstrated moderate $(2+)$ intensity staining. In the transition zone, epithelium in benign prostatic hyperplastic nodules demonstrated stronger staining compared with nonatrophic glands in the peripheral zone or central zones. Atrophic glands occasionally demonstrated stronger ( 1 to $2+$ ) staining than that seen in immediately adjacent glands without atrophy. In addition to epithelial staining, stromal cells in the transition zone typically demonstrated 1 to $2+$ staining, especially in nodular hyperplasia.

\section{Ki-67 Labeling Index of the Invasive Fronts and Central Bulk}

Among 15 selected cases with a pronounced difference in SNPH scores between the invasive front (SNPH expression $2+$ to $3+$ ) and central tumor bulk (SNPH expression 0 to $1+)$, the Ki-67 LI was higher at the invasive front (5\% versus $3.7 \% ; P=0.011$ ).

\section{RNA Expression in Cancer and Benign Peripheral Zone Tissue}

Recent studies have demonstrated that transcription of the $S N P H$ locus in humans produces at least two alternatively spliced isoforms: a long SNPH variant, corresponding to previously described neuronal $S N P H$; and a short, extraneuronal $S N P H$ isoform, characterized by mitochondrial localization and functionally implicated in the balance between tumor cell proliferation and tumor cell invasion. ${ }^{16}$ Quantitative PCR of individual SNPH isoforms in fresh benign and tumor samples identified the short $S N P H$ isoform as the dominant variant expressed in these settings (Figure 7A). In contrast, a long $S N P H$ isoform, previously

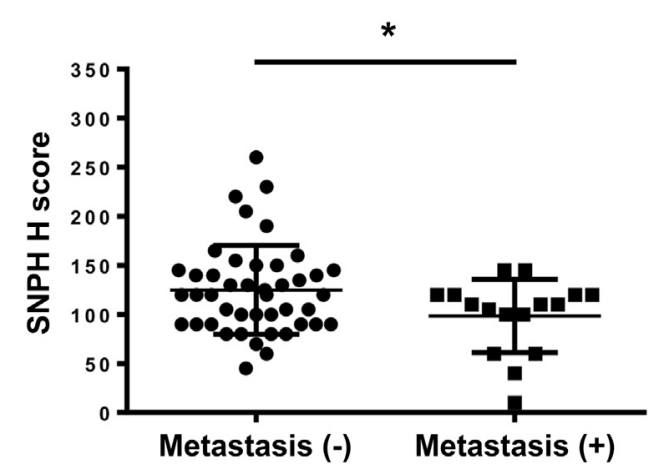

Figure 5 SNPH H scores in central tumor bulks are lower in grade group 2 to 5 cases with metastases than in cases without metastases. ${ }^{*} P<0.05$. 

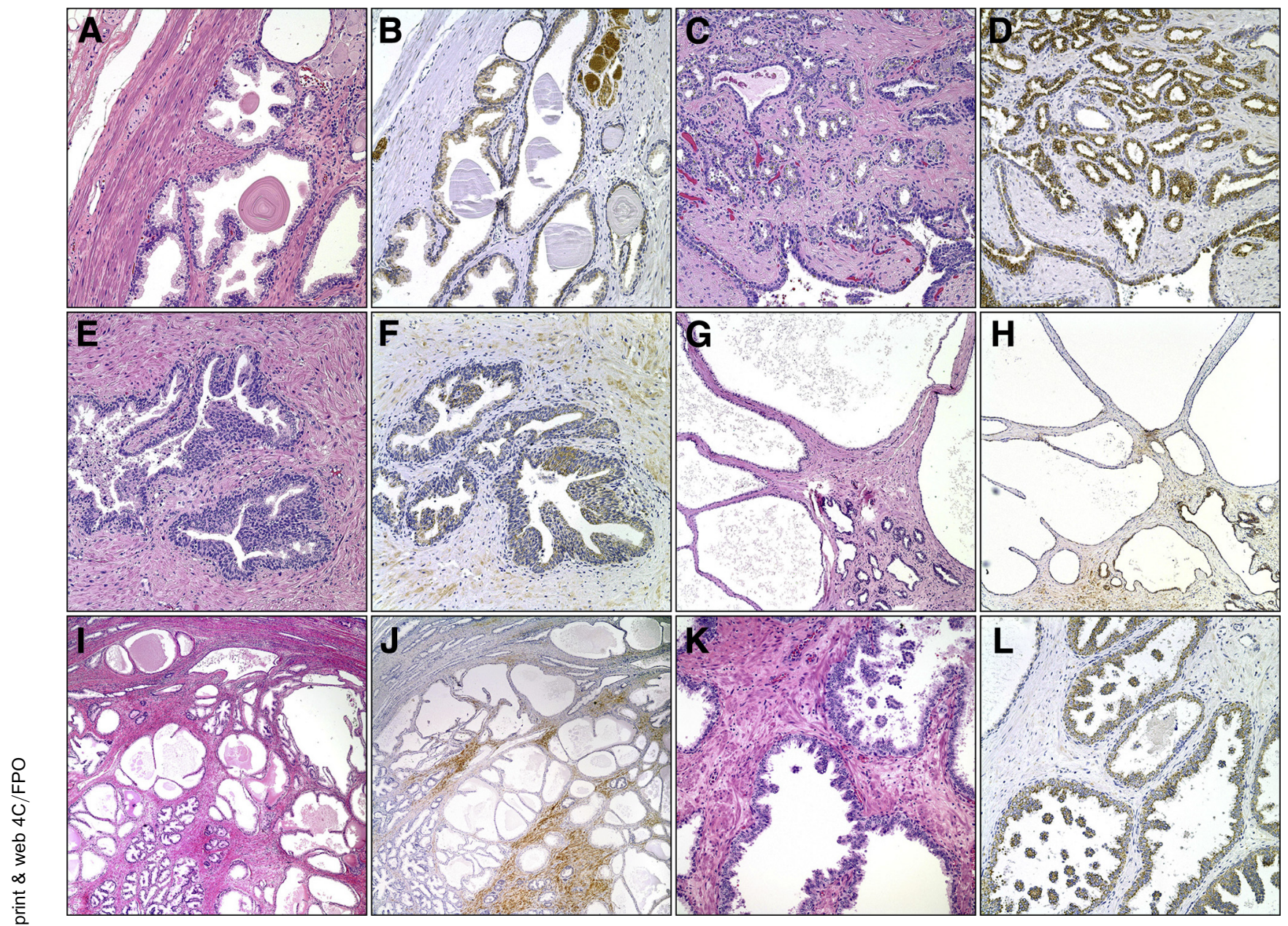

Figure 6 Hematoxylin and eosin (H\&E)-stained slides and corresponding SNPH immunostains in normal structures and nonmalignant conditions of the prostate. A and B: Peripheral zone acini showing 1+ SNPH expression. Ganglion cells and nerve bundles are recognized and show $2+$ to $3+$ staining. C and D: Seminal vesicle with clusters of acini and ducts, demonstrating intracytoplasmic lipofuscin pigment on H\&E-stained slides. The epithelial cells show heterogeneous SNPH intensity, ranging from $1+$ to $3+$, with a trend of weaker positivity in the ducts. E and F: Urothelial metaplasia with heterogeneous $1+$ to $2+$ SNPH staining. $\mathbf{G}$ and $\mathbf{H}$ : Simple and partial atrophy, with flat to low-columnar epithelial cells demonstrating decreased cytoplasmic volume. The epithelial cells show variable $1+$ to $2+$ positivity, with higher staining identified in partial atrophy at the right lower corner. I and J: Benign prostatic hyperplasia, with stromal cells demonstrating $2+$ SNPH staining. The stromal cells of the peripheral zone show focal weak 1+ positivity. K and L: High-grade prostatic intraepithelial neoplasia with a micropapillary pattern and a tufting pattern. The luminal cells show prominent nucleoli and stronger $1+$ to $2+$ staining compared with the benign acini, with 0 to $1+$ staining at the left upper corner. Original magnification: $\times 200(\mathbf{A}-\mathbf{F}, \mathbf{K}$, and $\mathbf{L}) ; \times 100(\mathbf{G}$ and $\mathbf{H}) ; \times 40$ (I and $\mathbf{J})$.

characterized for expression in neuronal tissue, was mostly undetectable in benign prostate and primary tumor samples (Figure $7 \mathrm{~A}$ ). ${ }^{16}$ In addition, there was a preliminary trend for higher expression of short $S N P H$ in tumor, compared with normal prostate samples (Figure 7B).

\section{Discussion}

In this study, SNPH, a molecule originally described as neuronal specific, was found to be abundantly expressed in prostate cancer, where its levels correlated with higher Gleason grade tumors. A distinctive feature of these observations is that SNPH was expressed in prostate cancer with a spatially unique distribution: higher SNPH levels were more prominently localized at the invasive front compared with the central tumor bulk, correlating with increased Ki-67 labeling also at the invasive front. In addition, the lower SNPH levels in the central tumor bulk were further down-regulated in patients with metastatic disease. Using isoform-specific molecular analysis, it was found that prostate cancer expresses a novel, short SNPH variant localized to mitochondria and functionally implicated in controlling the balance between cell proliferation and cell invasion.

The molecular and cellular determinants of metastatic prostate cancer have not been completely elucidated, but there is growing consensus that progressing disease is clonally heterogeneous, which may select for cells with endowed metastatic propensity. ${ }^{6-8}$ The results presented herein of a heterogeneous, location-specific expression of SNPH may be consistent with this model, potentially 

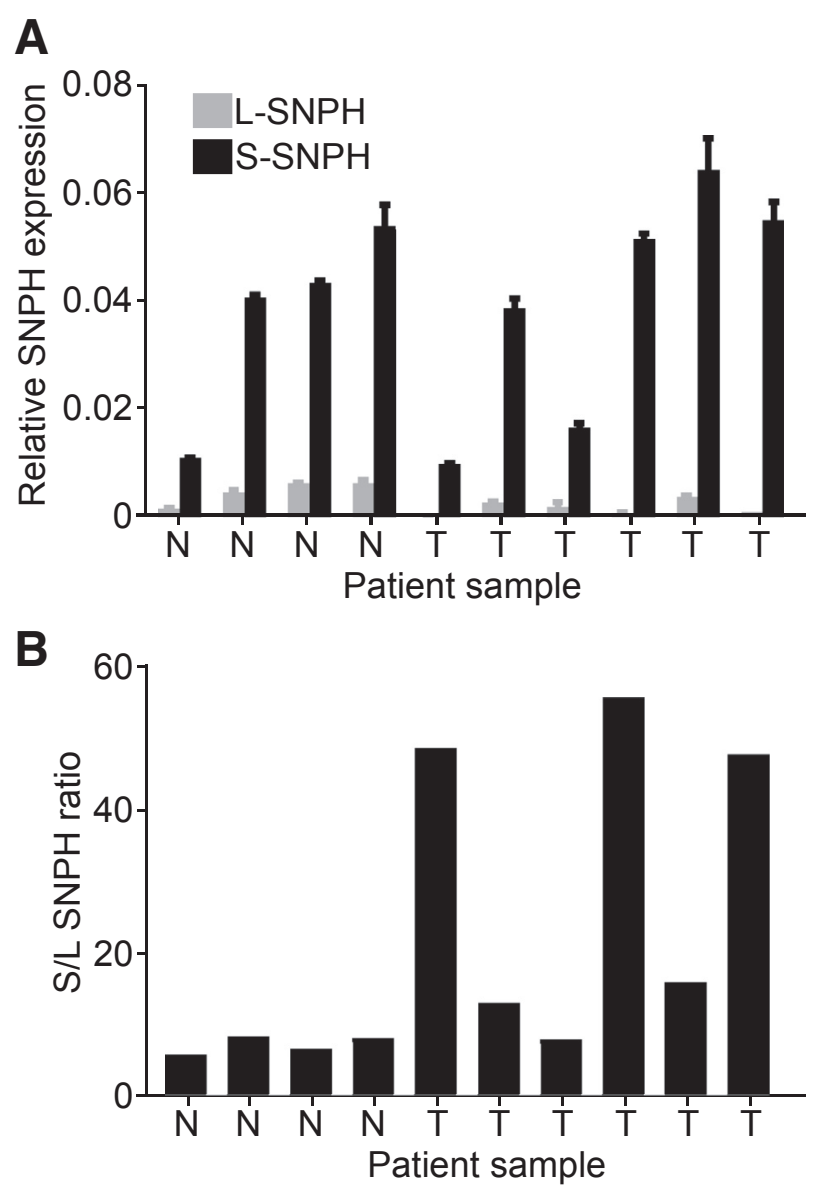

Figure 7 A: Total RNA extracted from the indicated prostate tissue samples was amplified with isoform-specific primers for the detection of a long (L) or short (S) SNPH isoform by $\beta$-actin-normalized quantitative PCR. B: The conditions are as in $\mathbf{A}$, and the ratio of S/L SNPH expression in normal $(\mathrm{N})$ or tumor $(\mathrm{T})$ prostate tissue samples was calculated. Data are expressed as means \pm SD of replicates (A). $n=2$ independent amplification experiments $(\mathbf{A})$.

identifying malignant clones with differential proliferative or migratory potential. In most cancer nodules, strong SNPH staining was limited to a few layers of cells at the outer tumor-stroma interface, which was defined as the invasive front, whereas the cells internal to the invasive front (central tumor bulk) showed weak to negative SNPH staining in most cases. This degree of heterogeneity has been observed before in prostate cancer and may reflect differences in nutrient availability or other stress conditions of the tumor microenvironment. Accordingly, Sethi et $\mathrm{al}^{26}$ demonstrated that proteins involved in the epithelialmesenchymal transition are more prominently expressed at the invasive front in human prostate cancer. In addition, Esposito et $\mathrm{al}^{27}$ also demonstrated stronger staining of SNAI2, a zinc-finger protein of the Snail family of transcription factors, at the invasive front, compared with the tumor center, potentially reflecting a differential methylation status of the SNAI2 gene promoter in the center of tumors versus the invasive front.
In addition to differences in spatial distribution, there was more pronounced expression of SNPH at the invasive front in GG 2 to 5 compared with GG 1 cases and a trend of increased SNPH expression in adenocarcinoma of higher Gleason grade. These findings suggest a positive correlation between SNPH expression at the invasive front and aggressive disease, in agreement with a small, but statistically significant, increase in the Ki-67 LI at the invasive front compared with the central tumor bulk. The idea of increased cell proliferation at the invasive front in prostate cancer is not widely studied, but there is evidence for high $\mathrm{Ki}-67$ expression at the invasive front in breast cancer, ${ }^{28}$ correlating with postoperative distant metastasis and disease-specific survival. ${ }^{29}$ This may be consistent with laboratory findings in which a three-dimensional tumoroid culture of prostate cancer cell lines also demonstrated increased Ki-67 staining at the invasive front. ${ }^{30}$ Conversely, analysis of the central tumor bulk showed almost invariably lower $\mathrm{H}$ scores for SNPH than those at the invasive front of the same tumor. When GG 2 to 5 cases were further subcategorized into those with or without metastasis, cases with disseminated disease showed weaker SNPH at the central tumor bulk compared with cases without metastasis. The reduced level of SNPH expression in these settings is in keeping with recent evidence obtained in cancer cell lines and prostate cancer xenograft models, in which loss of SNPH was associated with increased tumor cell migration, invasion, and metastatic dissemination. ${ }^{14,16}$

In addition to a positive correlation between $\mathrm{Ki}-67 \mathrm{LI}$ and SNPH expression, it was found that pure GP 3 glands had lower SNPH expression than GP 3 glands associated with GP 4 or 5. This observation fits well with the prior work in which pure GP 3 adenocarcinoma glands had a lower Ki-67 LI than GP 3 glands associated with cribriform GP $4 .{ }^{31}$ In fact, a potential biological difference between glands of pure GP 3 and GP 3 associated with GP 4 or 5 has been previously proposed. One study demonstrated that phosphatase and tensin homolog (PTEN) loss, $L P L / 8 \mathrm{p}$ loss, and $M Y C / 8 \mathrm{q}$ gain were more commonly identified in GP 3 glands associated with GP 4 than pure GP 3 glands. ${ }^{32}$ Furthermore, it has been shown that hundreds of genes are differently expressed in GP 3 glands embedded in GS 7 prostatic adenocarcinoma compared with pure GP 3 glands in GS 6 prostatic adenocarcinoma. ${ }^{33}$ Independent studies have also demonstrated a clonal relationship between GP 3 glands and the adjacent adenocarcinoma glands of higher Gleason grade in at least a subset of cases. ${ }^{34,35}$ However, GP 3 and associated GP 4 glands likely diverge early during cancer progression, given that there are multiple private mutations that are not shared between them. Interestingly, GP 3 glands in these cases retain the typical indolent-appearing histologic pattern, although they have acquired multiple additional molecular alterations, in addition to the shared genetic alterations with adjacent GP 4 glands. ${ }^{35}$ Haffner et $\mathrm{al}^{36}$ reported a case in which the lethal clone from a metastasis was traced back to a small region in the primary tumor, composed of GP 3 glands exhibiting loss 
of PTEN expression, an SPOP mutation, and a TP53 mutation. Interestingly, the molecular changes of those lethal metastases suggest that they did not arise from tumor glands of higher Gleason grade near the GP 3 region. This suggests that cancer of low-grade morphology may still be the origin of a lethal clone when it is embedded in glands of higher Gleason grade. Our SNPH data, revealing differences in GP 3 expression between GG 1 and GG 2 to 5 cases, provide additional evidence that not all GP 3 glands are identical. Whether SNPH expression has a potential role as a biomarker for identifying higher-risk GP 3 tumor glands will require further study.

Taken together, the results presented herein can be explained by the dual function of SNPH in cancer. Consistent with recent findings, we demonstrated that a short, extraneuronal $S N P H$ variant was the dominant form expressed in our series of prostate cancer. ${ }^{16}$ At the molecular level, this SNPH isoform localizes to mitochondria and maintains oxidative bioenergetics, supporting ATP production and reduced generation of toxic reactive oxygen species. Accordingly, high SNPH levels supported increased tumor cell proliferation, ${ }^{14,16}$ in line with the observations reported herein that high SNPH expression at the invasive front correlated with an increased Ki-67 LI. Likewise, the observed SNPH expression in distant metastases may indicate that these lesions remain highly proliferative. Conversely, reduction of SNPH levels reduced proliferation but dramatically enhanced tumor cell motility. This was attributable to the second function of SNPH as an inhibitor of mitochondrial trafficking. Accordingly, loss of SNPH in these settings promoted heightened redistribution of mitochondria to the cortical cytoskeleton of tumor cells, fueling increased membrane dynamics of cell motility, tumor cell invasion, and metastasis in vivo. ${ }^{16}$ This dual function of SNPH in controlling the balance between cell proliferation and cell motility, a process also called phenotype switching, ${ }^{37}$ may explain the biphasic spatial distribution of SNPH observed herein. Accordingly, evidence collected from cell culture models, including prostate cancer, ${ }^{16}$ suggests that SNPH levels are exquisitely sensitive to changes commonly observed in the microenvironment of tumor growth. In this context, microenvironment stress conditions in the central tumor bulk due to hypoxia or reduced nutrient availability may result in SNPH loss and increased metastatic propensity, whereas more favorable conditions of unrestricted oxygen and nutrient availability at the invasive front may maintain higher levels of SNPH in the tumor cell population, resulting in sustained mitochondrial bioenergetics and $\mathrm{Ki}-67^{+}$tumor cell proliferation. Consistent with this model, hypoxia, as quantified by direct electrode measurement and immunohistochemical studies, ${ }^{38,39}$ has long been recognized as a key driver of prostate cancer, influencing protein expression, ${ }^{40}$ intraductal carcinoma/cribriform architecture ${ }^{41}$ higher Gleason scores and grades, ${ }^{42}$ a more aggressive clinical stage, ${ }^{43}$ and increased incidence of biochemical recurrence. $^{44}$
In summary, we have identified a unique, spatially biphasic distribution of SNPH in prostate cancer with sharp differential expression between the invasive front and central bulk regions of the tumor. Broadly, these findings may reinforce a proposed model of clonal heterogeneity of progressing prostate cancer and suggest that SNPH may be differentially exploited in central versus proliferative sites, depending on the conditions of the tumor microenvironment. The results obtained herein in primary patient cohorts also reaffirm a critical, emerging role of mitochondrial biology in influencing tumor behavior, including prostate cancer. Whether a spatially differential expression of SNPH could help identify patients with greater risk of metastatic dissemination is worthy of further investigation.

\section{Supplemental Data}

Supplemental material for this article can be found at http://doi.org/10.1016/j.ajpath.2019.02.009.

\section{References}

1. Cronin KA, Lake AJ, Scott S, Sherman RL, Noone AM, Howlader N Henley SJ, Anderson RN, Firth AU, Ma J, Kohler BA, Jemal A: Annual Report to the Nation on the Status of Cancer, part I: national cancer statistics. Cancer 2018, 124:2785-2800

2. Litwin MS, Tan HJ: The diagnosis and treatment of prostate cancer: a review. JAMA 2017, 317:2532-2542

3. Siegel RL, Miller KD, Jemal A: Cancer statistics, 2017. CA Cancer J Clin 2017, 67:7-30

4. Berish RB, Ali AN, Telmer PG, Ronald JA, Leong HS: Translational models of prostate cancer bone metastasis. Nat Rev Urol 2018, 15:403-421

5. Liu W, Laitinen S, Khan S, Vihinen M, Kowalski J, Yu G, Chen L, Ewing CM, Eisenberger MA, Carducci MA, Nelson WG, Yegnasubramanian S, Luo J, Wang Y, Xu J, Isaacs WB, Visakorpi T, Bova GS: Copy number analysis indicates monoclonal origin of lethal metastatic prostate cancer. Nat Med 2009, 15:559-565

6. Gundem G, Van Loo P, Kremeyer B, Alexandrov LB, Tubio JMC Papaemmanuil E, Brewer DS, Kallio HML, Hognas G, Annala M, Kivinummi K, Goody V, Latimer C, O’Meara S, Dawson KJ, Isaacs W, Emmert-Buck MR, Nykter M, Foster C, Kote-Jarai Z, Easton D, Whitaker HC, Group IP, Neal DE, Cooper CS, Eeles RA, Visakorpi T, Campbell PJ, McDermott U, Wedge DC, Bova GS: The evolutionary history of lethal metastatic prostate cancer. Nature 2015, 520:353-357

7. Hong MK, Macintyre G, Wedge DC, Van Loo P, Patel K, Lunke S, Alexandrov LB, Sloggett C, Cmero M, Marass F, Tsui D, Mangiola S, Lonie A, Naeem H, Sapre N, Phal PM, Kurganovs N, Chin X, Kerger M, Warren AY, Neal D, Gnanapragasam V, Rosenfeld N, Pedersen JS, Ryan A, Haviv I, Costello AJ, Corcoran NM, Hovens CM: Tracking the origins and drivers of subclonal metastatic expansion in prostate cancer. Nat Commun 2015, 6:6605

8. Van Etten JL, Dehm SM: Clonal origin and spread of metastatic prostate cancer. Endocr Relat Cancer 2016, 23:R207-R217

9. Vander Heiden MG, Cantley LC, Thompson CB: Understanding the Warburg effect: the metabolic requirements of cell proliferation. Science 2009, 324:1029-1033

10. Altieri DC: Mitochondria on the move: emerging paradigms of organelle trafficking in tumour plasticity and metastasis. Br J Cancer 2017, 117:301-305

11. Roesch A, Vultur A, Bogeski I, Wang H, Zimmermann KM, Speicher D, Korbel C, Laschke MW, Gimotty PA, Philipp SE, Krause E, Patzold S Villanueva J, Krepler C, Fukunaga-Kalabis M, Hoth M, Bastian BC, 
Vogt T, Herlyn M: Overcoming intrinsic multidrug resistance in melanoma by blocking the mitochondrial respiratory chain of slow-cycling JARID1B(high) cells. Cancer Cell 2013, 23:811-825

12. LeBleu VS, O'Connell JT, Gonzalez Herrera KN, Wikman H, Pantel K, Haigis MC, de Carvalho FM, Damascena A, Domingos Chinen LT, Rocha RM, Asara JM, Kalluri R: PGC-1alpha mediates mitochondrial biogenesis and oxidative phosphorylation in cancer cells to promote metastasis. Nat Cell Biol 2014, 16:992-1003. 1-15

13. Caino MC, Ghosh JC, Chae YC, Vaira V, Rivadeneira DB, Faversani A, Rampini P, Kossenkov AV, Aird KM, Zhang R, Webster MR, Weeraratna AT, Bosari S, Languino LR, Altieri DC: PI3K therapy reprograms mitochondrial trafficking to fuel tumor cell invasion. Proc Natl Acad Sci U S A 2015, 112:8638-8643

14. Caino MC, Seo JH, Aguinaldo A, Wait E, Bryant KG, Kossenkov AV, Hayden JE, Vaira V, Morotti A, Ferrero S, Bosari S, Gabrilovich DI, Languino LR, Cohen AR, Altieri DC: A neuronal network of mitochondrial dynamics regulates metastasis. Nat Commun 2016, 7:13730

15. Kang JS, Tian JH, Pan PY, Zald P, Li C, Deng C, Sheng ZH: Docking of axonal mitochondria by syntaphilin controls their mobility and affects short-term facilitation. Cell 2008, 132:137-148

16. Caino MC, Seo JH, Wang Y, Rivadeneira DB, Gabrilovich DI, Kim ET, Weeraratna AT, Languino LR, Altieri DC: Syntaphilin controls a mitochondrial rheostat for proliferation-motility decisions in cancer. J Clin Invest 2017, 127:3755-3769

17. Epstein JI, Egevad L, Amin MB, Delahunt B, Srigley JR, Humphrey PA, Grading C: The 2014 International Society of Urological Pathology (ISUP) Consensus Conference on Gleason Grading of Prostatic Carcinoma: definition of grading patterns and proposal for a new grading system. Am J Surg Pathol 2016, 40:244-252

18. IARC, Moch H: WHO Classification of Tumours of the Urinary System and Male Genital Organs. ed 4. Lyon, France, International Agency for Research on Cancer, 2016

19. Compton CC, Byrd DR, Garcia-Aguilar J, Kurtzman SH, Olawaiye A, Washington MK: AJCC Cancer Staging Atlas: A Companion to the Seventh Editions of the AJCC Cancer Staging Manual and Handbook. ed 2. New York: Springer, 2012

20. Iczkowski KA, Torkko KC, Kotnis GR, Wilson RS, Huang W, Wheeler TM, Abeyta AM, La Rosa FG, Cook S, Werahera PN, Lucia MS: Digital quantification of five high-grade prostate cancer patterns, including the cribriform pattern, and their association with adverse outcome. Am J Clin Pathol 2011, 136:98-107

21. Kweldam CF, Wildhagen MF, Steyerberg EW, Bangma CH, van der Kwast TH, van Leenders GJ: Cribriform growth is highly predictive for postoperative metastasis and disease-specific death in Gleason score 7 prostate cancer. Mod Pathol 2015, 28:457-464

22. Siadat F, Sykes J, Zlotta AR, Aldaoud N, Egawa S, Pushkar D, Kuk C, Bristow RG, Montironi R, van der Kwast T: Not all Gleason pattern 4 prostate cancers are created equal: a study of latent prostatic carcinomas in a cystoprostatectomy and autopsy series. Prostate 2015, 75:1277-1284

23. Iczkowski KA, Paner GP, Van der Kwast T: The new realization about cribriform prostate cancer. Adv Anat Pathol 2018, 25:31-37

24. Loeb S, Folkvaljon Y, Robinson D, Lissbrant IF, Egevad L, Stattin P: Evaluation of the 2015 Gleason grade groups in a nationwide population-based cohort. Eur Urol 2016, 69:1135-1141

25. Epstein JI, Zelefsky MJ, Sjoberg DD, Nelson JB, Egevad L, MagiGalluzzi C, Vickers AJ, Parwani AV, Reuter VE, Fine SW, Eastham JA, Wiklund P, Han M, Reddy CA, Ciezki JP, Nyberg T, Klein EA: A contemporary prostate cancer grading system: a validated alternative to the Gleason score. Eur Urol 2016, 69:428-435

26. Sethi S, Macoska J, Chen W, Sarkar FH: Molecular signature of epithelial-mesenchymal transition (EMT) in human prostate cancer bone metastasis. Am J Transl Res 2010, 3:90-99

27. Esposito S, Russo MV, Airoldi I, Tupone MG, Sorrentino C, Barbarito G, Di Meo S, Di Carlo E: SNAI2/Slug gene is silenced in prostate cancer and regulates neuroendocrine differentiation, metastasis-suppressor and pluripotency gene expression. Oncotarget 2015, 6:17121-17134
28. Lloyd MC, Cunningham JJ, Bui MM, Gillies RJ, Brown JS, Gatenby RA: Darwinian dynamics of intratumoral heterogeneity: not solely random mutations but also variable environmental selection forces. Cancer Res 2016, 76:3136-3144

29. Gong P, Wang Y, Liu G, Zhang J, Wang Z: New insight into Ki67 expression at the invasive front in breast cancer. PLoS One 2013, 8:e54912

30. Aw Yong KM, Li Z, Merajver SD, Fu J: Tracking the tumor invasion front using long-term fluidic tumoroid culture. Sci Rep 2017, 7:10784

31. Fu L, Hwang M, Adeniran AJ, Humphrey PA: Proliferation index of different Gleason pattern 4 histomorphologies and associated pattern 3 adenocarcinoma of the prostate. Hum Pathol 2017, 70:1-5

32. Trock BJ, Fedor H, Gurel B, Jenkins RB, Knudsen BS, Fine SW, Said JW, Carter HB, Lotan TL, De Marzo AM: PTEN loss and chromosome 8 alterations in Gleason grade 3 prostate cancer cores predicts the presence of un-sampled grade 4 tumor: implications for active surveillance. Mod Pathol 2016, 29:764-771

33. Hoogland AM, Bottcher R, Verhoef E, Jenster G, van Leenders GJ: Gene-expression analysis of Gleason grade 3 tumor glands embedded in low- and high-risk prostate cancer. Oncotarget 2016, 7:37846-37856

34. Kovtun IV, Cheville JC, Murphy SJ, Johnson SH, Zarei S, Kosari F, Sukov WR, Karnes RJ, Vasmatzis G: Lineage relationship of Gleason patterns in Gleason score 7 prostate cancer. Cancer Res 2013, 73: 3275-3284

35. Sowalsky AG, Kissick HT, Gerrin SJ, Schaefer RJ, Xia Z, Russo JW, Arredouani MS, Bubley GJ, Sanda MG, Li W, Ye H, Balk SP: Gleason score 7 prostate cancers emerge through branched evolution of clonal Gleason pattern 3 and 4. Clin Cancer Res 2017, 23:3823-3833

36. Haffner MC, Mosbruger T, Esopi DM, Fedor H, Heaphy CM, Walker DA, Adejola N, Gurel M, Hicks J, Meeker AK, Halushka MK, Simons JW, Isaacs WB, De Marzo AM, Nelson WG, Yegnasubramanian S: Tracking the clonal origin of lethal prostate cancer. J Clin Invest 2013, 123:4918-4922

37. Kemper K, de Goeje PL, Peeper DS, van Amerongen R: Phenotype switching: tumor cell plasticity as a resistance mechanism and target for therapy. Cancer Res 2014, 74:5937-5941

38. Movsas B, Chapman JD, Horwitz EM, Pinover WH, Greenberg RE, Hanlon AL, Iyer R, Hanks GE: Hypoxic regions exist in human prostate carcinoma. Urology 1999, 53:11-18

39. Rupp NJ, Schuffler PJ, Zhong Q, Falkner F, Rechsteiner M, Ruschoff JH, Fankhauser C, Drach M, Largo R, Tremp M, Poyet C, Sulser T, Kristiansen G, Moch H, Buhmann J, Muntener M, Wild PJ: Oxygen supply maps for hypoxic microenvironment visualization in prostate cancer. J Pathol Inform 2016, 7:3

40. Tonry C, Armstrong J, Pennington S: Probing the prostate tumour microenvironment II: impact of hypoxia on a cell model of prostate cancer progression. Oncotarget 2017, 8:15307-15337

41. Chua MLK, Lo W, Pintilie M, Murgic J, Lalonde E, Bhandari V, Mahamud O, Gopalan A, Kweldam CF, van Leenders G, Verhoef EI, Hoogland AM, Livingstone J, Berlin A, Dal Pra A, Meng A, Zhang J, Orain M, Picard V, Hovington H, Bergeron A, Lacombe L, Fradet Y, Tetu B, Reuter VE, Fleshner N, Fraser M, Boutros PC, van der Kwast TH, Bristow RG: A prostate cancer "Nimbosus": genomic instability and SChLAP1 dysregulation underpin aggression of intraductal and cribriform subpathologies. Eur Urol 2017, 72:665-674

42. Ambrosio MR, Di Serio C, Danza G, Rocca BJ, Ginori A, Prudovsky I, Marchionni N, Del Vecchio MT, Tarantini F: Carbonic anhydrase IX is a marker of hypoxia and correlates with higher Gleason scores and ISUP grading in prostate cancer. Diagn Pathol 2016, 11:45

43. Movsas B, Chapman JD, Greenberg RE, Hanlon AL, Horwitz EM, Pinover WH, Stobbe C, Hanks GE: Increasing levels of hypoxia in prostate carcinoma correlate significantly with increasing clinical stage and patient age: an Eppendorf pO(2) study. Cancer 2000, 89:2018-2024

44. Lalonde E, Ishkanian AS, Sykes J, Fraser M, Ross-Adams H, Erho N, et al: Tumour genomic and microenvironmental heterogeneity for integrated prediction of 5-year biochemical recurrence of prostate cancer: a retrospective cohort study. Lancet Oncol 2014, 15: $1521-1532$ 